\title{
Wearable Gaze Trackers Mapping Visual Attention in 3D
}

Jensen, Rasmus; Stets, Jonathan Dyssel; Suurmets, Seidi; Clement, Jesper; Aanæs, Henrik

Document Version

Accepted author manuscript

Published in:

Scandinavian Conference on Image Analysis

DOI:

10.1007/978-3-319-59126-1_6

Publication date:

2017

License

Unspecified

Citation for published version (APA):

Jensen, R., Stets, J. D., Suurmets, S., Clement, J., \& Aanæs, H. (2017). Wearable Gaze Trackers: Mapping

Visual Attention in 3D. In P. Sharma, \& F. M. Bianchi (Eds.), Scandinavian Conference on Image Analysis: 20th Scandinavian Conference, SCIA 2017 Tromsø, Norway, June 12-14, 2017 Proceedings, Part I (pp. 66-76). Springer. Lecture Notes in Computer Science Vol. 10269 https://doi.org/10.1007/978-3-319-59126-1_6

Link to publication in CBS Research Portal

\section{General rights}

Copyright and moral rights for the publications made accessible in the public portal are retained by the authors and/or other copyright owners and it is a condition of accessing publications that users recognise and abide by the legal requirements associated with these rights.

Take down policy

If you believe that this document breaches copyright please contact us (research.lib@cbs.dk) providing details, and we will remove access to the work immediately and investigate your claim. 


\section{Wearable Gaze Trackers: Mapping Visual Attention in 30 \\ Rasmus Jensen, Jonathan Dyssel Stets, Seidi Suurmets, Jesper Clement, and Henrik Aanæs}

Journal article (Accepted version)

CITE: Wearable Gaze Trackers : Mapping Visual Attention in 3D. / Jensen, Rasmus; Stets, Jonathan Dyssel; Suurmets, Seidi; Clement, Jesper; Aanæs, Henrik. In: Scandinavian Conference on Image Analysis: 20th Scandinavian Conference, SCIA 2017 Tromso, Norway, June 12-14, 2017 Proceedings, Part I. ed. / Puneet Sharma; Filippo Maria Bianchi. Cham : Springer, 2017. p. $66-76$.

This is a post-peer-review, pre-copyedit version of an article published in Scandinavian Conference on Image Analysis: 20th Scandinavian Conference, SCIA 2017 Troms, Norway, June 12-14, 2017 Proceedings, Part I.

The final authenticated version is available online at: https://doi.org/10.1007/978-

3-319-59126-1_6

Uploaded to CBS Research Portal: January २०19 


\title{
Wearable Gaze Trackers: Mapping Visual Attention in 3D
}

\author{
Rasmus R. Jensen ${ }^{1}$, Jonathan D. Stets ${ }^{1}$, Seidi Suurmets ${ }^{2}$, Jesper Clement ${ }^{2}$, \\ and Henrik Aanæs ${ }^{1}$ \\ 1 Technical University of Denmark \\ 2 Copenhagen Business School
}

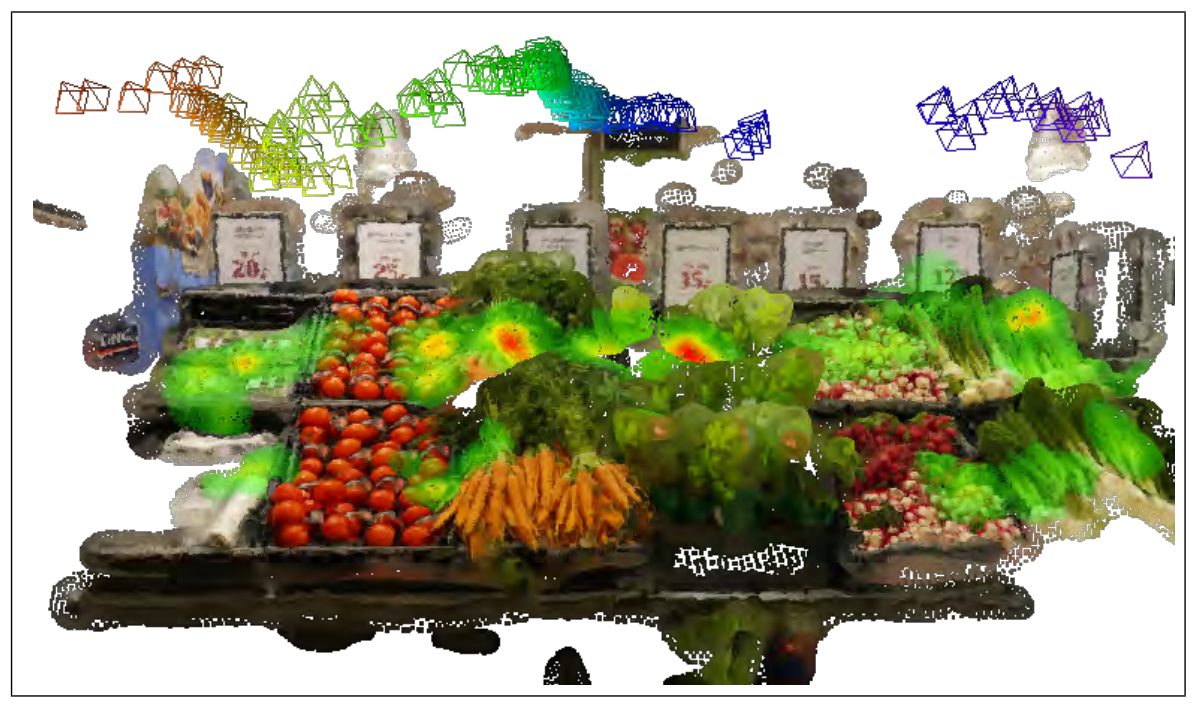

Fig. 1. Supermarket vegetables shown as a 3D model with heatmap and respondent viewing points.

\begin{abstract}
The study of visual attention in humans relates to a wide range of areas such as: psychology, cognition, usability, and marketing. These studies have been limited to fixed setups with respondents sitting in front of a monitor mounted with a gaze tracking device. The introduction of wearable mobile gaze trackers allows respondents to move freely in any real world $3 \mathrm{D}$ environment, removing the previous restrictions. In this paper we propose a novel approach for processing visual attention of respondents using mobile wearable gaze trackers in a $3 \mathrm{D}$ environment. The pipeline consists of 3 steps: modeling the 3D area-of-interest, positioning the gaze tracker in 3D space, and 3D mapping of visual attention. The approach is general, but as a case study we have used it to create 3D heatmaps of respondents visiting supermarket shelves as well as their instore movement relative to these shelves. The method allows for analysis
\end{abstract}


across multiple respondents and to distinguish between phases of in-store orientation (far away) and product recognition/selection (up close) based on distance to shelves.

\section{Introduction}

The study of human visual attention relates to a wide range of areas such as: psychology, cognition, useability, and marketing. In order to directly study this in various settings, eye tracking has become a standard method. A common way of visualizing and analysing gaze data is using Areas Of Interest (AOI) and attentional heat maps [11]. The heat maps represent the spatial distribution of eye movement throughout the AOI and can often be used for quantitative analysis. The most common method of visualizing heat maps is using a Gaussian based solution. Here, four parameters are used to determine the appearance of the heat map: the width of the basic construct, the use of fixations vs.

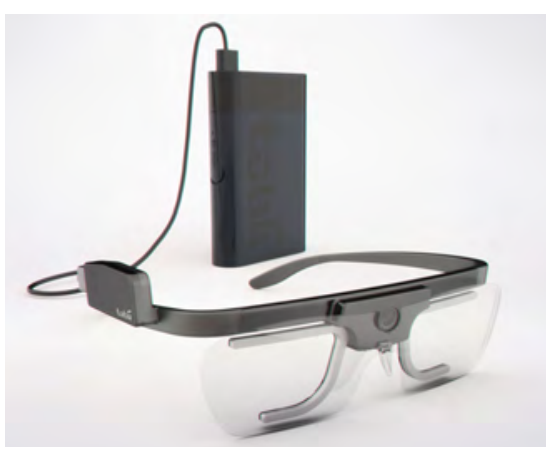

Fig. 2. Tobii Pro Glasses 2 [10]. A wearable eye tracker that tracks a respondents eye movements using IR cameras, while also recording the environment with a front facing video camera. raw data, whether accounting for fixation duration and the mapping color altitude form [3].

For many years, mapping visual attention as heat maps has been limited to static setups with respondents sitting in front of a screen mounted with a stationary calibrated gaze tracker. Such a setup can accurately map the visual attention as a heat map of what is projected on the screen, but obviously limits the visual attention to a $2 \mathrm{D}$ surface. The recent introduction of mobile wearable gaze trackers (fig. 2) enables data collection in about any real-world environment. On mobile wearable eye-trackers, the scene is recorded using a front facing camera, and gaze data collected from eye tracking cameras can be projected onto this video. Despite the potential of introducing recordings of three dimensional scenes, common for both the stationary and mobile wearable eye-tracker is that ultimately the data is still recorded and analysed in $2 \mathrm{D}$.

Mapping visual attention data recorded in a $3 \mathrm{D}$ space to a $2 \mathrm{D}$ heatmap is not straight forward. A simple approach is to find the best homographic correspondence between a reference image and a given frame from the eye tracker, and then map the gaze according to this homography [4, 10]. Figure 3 shows common errors in mapping using a homography relative to the actual mapping onto a 3D AOI. We argue that gaze collected in 3D mapped onto a $2 \mathrm{D}$ reference image using a homography will always be limited as a result of incorrect mappings.

We propose a solution to these problems and limitations by modelling an $\mathrm{AOI}$ in $3 \mathrm{D}$ as a reference for mapping gaze data. The reference model is reconstructed from photographs of the AOI to establish a good base for image feature 

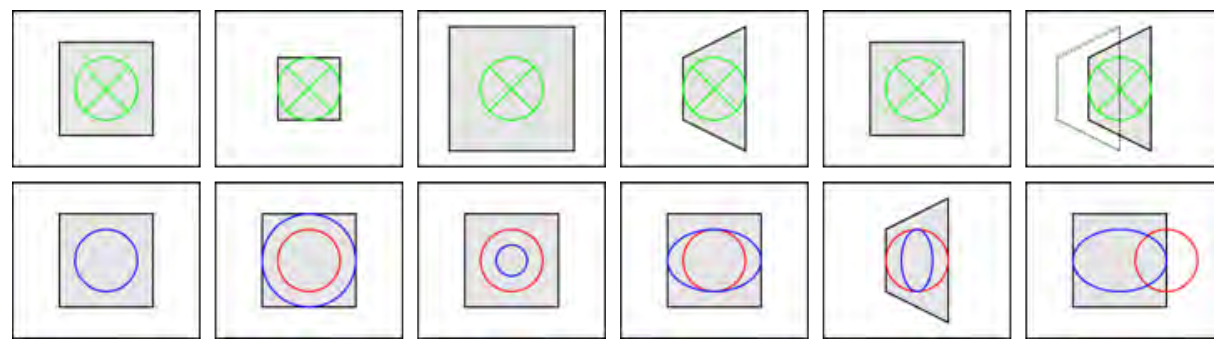

Fig. 3. This figure shows the common errors relating to mapping gaze as a shown as a circle based a homography between the viewpoint and a reference view. The top row shows the viewpoint of the respondent with the gaze point in green, while the second row shows a reference view of the AOI with gaze mapped as homography mapping in red and mapping according to 3D structure in blue. First column shows mapping, when the viewpoint of the respondent and reference coincide. In this case, homography mapping and mapping according to 3D structure will be identical and perfectly overlapping. Column 2 and 3 show the mapping, when respondent is closer to or further from the AOI. The homography mapping incorrectly does not change along with the movement of the respondent. Row 4 and 5 show how the gaze circle changes shape according to changes in viewpoint for the $3 \mathrm{D}$ mapping, while homography does not change as it should. The final column shows the error, when the homography is offset from the plane of the actual viewpoint, which introduces parallax error.

matching and a high quality model mesh. We demonstrate a fully automatic pipeline for generating a 3D attention heat map, and furthermore the possibility of calculating the respondent viewing points as shown in Figure 1. Our pipeline enables spatial filtering, positioning and orientation relative to the selected AOI, as well as correlation of multi respondent data. We use supermarket shelves as a case study, but our pipeline is not limited to this setup.

There are a number of recent studies that addresses the need for being able to move mapping of visual attention to 3D. [9] introduces the potential of measuring 3D gaze coordinates from head-mounted gaze trackers, and [7] proposes visualisation of $3 \mathrm{D}$ gaze data on to virtual computer generated models. A method similar to our pipeline is described in [8], which demonstrates the use of a Microsoft Kinect to create a 3D reference model. Our method differs by using images to create a more dense point cloud, which also enables us to backproject the heat map to a traditional $2 \mathrm{D}$ visualization for comparison.

\section{Data}

We have collected data in both a real world supermarket and using a mock-up supermarket shelf in our lab. Reference data of the AOIs have been captured using a digital mirrorless camera: a Panasonic GH4 with a $12 \mathrm{~mm}$ lens $(24 \mathrm{~mm}$ in $35 \mathrm{~mm}$ equivalent). To collect respondent data we have used the Tobii Pro Glasses 2 wearable gaze tracker [10] (fig. 2), which collects the respondents view using 


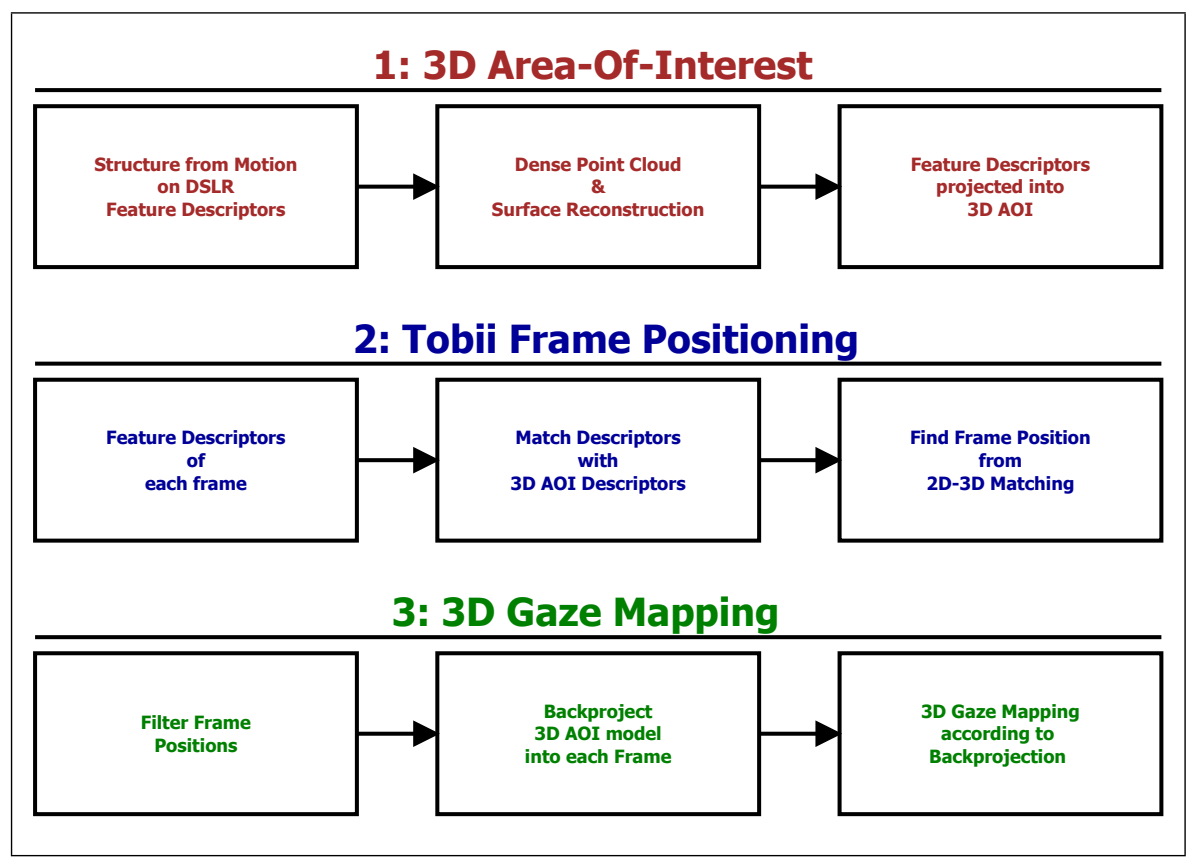

Fig. 4. The 3 steps in our proposed pipeline to construct 3D gazemaping: Modelling of an Area-Of-Interest, Eye-tracker frame positioning, and finally the gaze mapping.

a front facing video camera, while also recording the respondent gaze direction using 4 infrared cameras facing the eyes. Both cameras were calibrated using a standard checkerboard approach [14]. Data was collected of four in-store product sections in a supermarket: wine, vegetables, flour and cereal, as well as a mockup of the cereal section in our lab. We used the digital camera to capture sets of reference images to cover the desired AOIs (12-20 images of each AOI). Gaze and video data were collected of respondents visiting the given sections (16 sets), visiting the store but acquired to get cereal (4 sets), and finally, presented for a mock-up of the cereal section in the lab (6 sets). All gaze data samples are raw, so no fixation filtering has been applied [3].

\section{Method}

In order to map gaze data onto a 3D AOI, we propose a pipeline consisting of three parts (fig. 4): construction of the 3D AOI reference model, localization of the wearable gaze tracker frames relative to the reference model, and finally gaze mapping onto the AOI as a heat map. 


\subsection{Modelling a 3D Area-Of-Interest}

The 3D AOI reference model is built using a series of images of the AOI. As shown in (fig. 4), this task is divided into further three steps. First, we use structure from motion to find the spatial camera positions and a sparse point cloud representation. We have opted for a structure from motion (SfM) implementation, which requires a sequence of images followed by an image rectification based on the paramters obtained from the camera calibration. SIFT descriptors $[6,13]$ are found in each image and sequentially matched across the sequence of images in an iterative fashion. Images with sufficient feature matches are included, while the extrinsic camera parameters are estimated and refined using bundle adjustment [12].

Given the estimated extrinsic camera parameters, we move onto dense point cloud estimation using the patch-expansion approach to multiview stereopsis proposed by Furukawa and Ponce [2]. This method robustly produces dense representations from which a surface is reconstructed using Poisson surface reconstruction by Kazhdan et al. [5]. A 3D modelled AOI from the cereal section in a supermarket is shown in fig. 5(a).

As a preparation step for the localization of the wearable gaze tracker later in the pipeline, we use backprojection with depth management of the 3D AOI into each reference image. This is in order to project 2D SIFT descriptors [6] into the $3 \mathrm{D}$ space, allowing the $2 \mathrm{D}$ descriptors between each frame from the gaze tracker and 3D AOI to be compared.

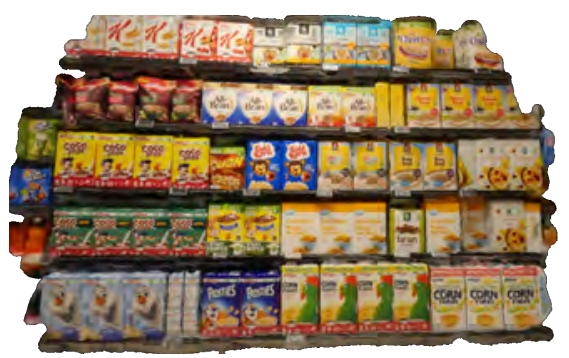

(a) 3D Area-Of-Interrest reference model.

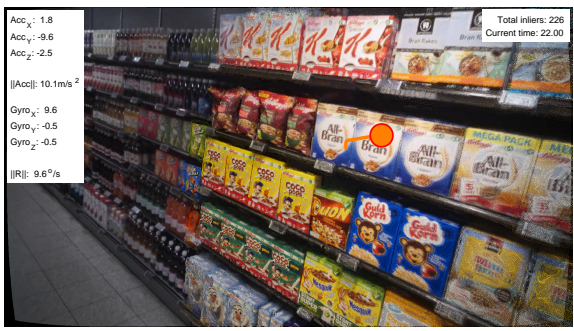

(b) The 3D AOI backprojected onto a gaze tracker frame.

Fig. 5. The 3D AOI from (a) is backprojected onto an undistorted gaze tracker frame and the gaze point with trace from previous frames (b).

\subsection{Wearable gaze tracker frame localization}

In order to correctly map gaze data on the 3D AOI, each frame from the gaze tracker has to be positioned relative to the AOI (if visible). The SIFT descriptors in each frame are matched with the reference $2 \mathrm{D}$ descriptors projected into 3D 
space, when constructing the 3D AOI. Since a given frame might not cover any part of the AOI, the resulting matching consists of either true positive or false positive matches. Without discriminating between true- or false positive, the correspondences are sent to a $2 \mathrm{D}$ to $3 \mathrm{D}$ pose solver, to find the best fit camera pose using a RANSAC approach discarding outliers [1]. Given sufficient corresponding points, the solver will return the correct camera pose relative to the AOI. Without sufficient good matches the solver either fails or returns a false camera pose. Figure 5(b) shows the 3D AOI backprojected into a frame from the wearable tracker using the estimated camera pose. This backprojection is an immediate sanity check, showing the correctness of the pose estimation. Incorrect pose estimates tends to be very inconsistent from one frame to the next.

\section{3 $\quad$ Mapping gaze data}

The pose estimation provides an unbiased set of output poses, and filtering will help to remove incorrect ones. Correct pose estimates between consecutive frames will vary very little, while incorrect poses are very inconsistent. This inconsistency is used to incorrect discard poses. Figure 6 shows the good pose estimates as a connected graphs with discarded poses as outlying points. The number of inliers returned from the 2D to $3 \mathrm{D}$ pose solver is a good estimator of correctness, but thresholding this number is not as robust as filtering the pose.
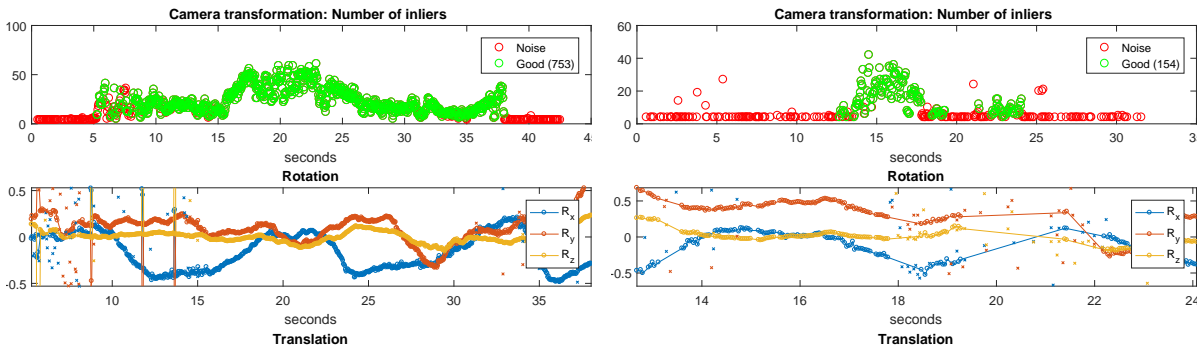

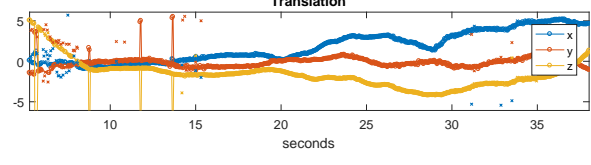

(a) Cereal shelves

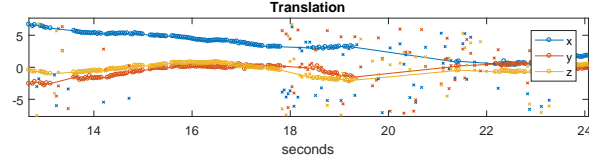

(b) Flour shelves

Fig. 6. Estimated poses for cereal and flour shelves. From top to bottom: the framewise number of inliers in the camera positioning solver, where green points are considered reliable and red points is noise. Second and third plot is the rotation and translation with inliers shown as a connected graph and outliers as single points.

Figure 7(a) shows a respondent moving in front of the AOI. Using the good poses, the gaze can be mapped onto the $3 \mathrm{D}$ AOI model creating a $3 \mathrm{D}$ heat 
map as seen in fig. 7(b). The gaze intersection with the 3D model is found using backprojection with depth management into the current frame, which is significantly faster than calculating the intersection between the line of sight and the 3D model.

A similar approach is taken, when creating the heatmap. Here a predetermined symmetric 2D Gaussian function with center at the gaze coordinate is added to a sum map of Gaussians in 3D space. Using a Gaussian serves both the fact that sight is not an infinitely small point, while also incorporating some uncertainty in the gaze estimates. Discussions about the size of the Gaussian, and whether the raw gaze data or fixation filtered data should be used is beyond the scope of this work. The resulting heatmaps visualized on the 3D AOIs can be seen in figs. 1 and $7(\mathrm{c})$ to $7(\mathrm{e})$.

One benefit worth noticing is, that the approach of mapping Gaussian to the backprojection of the AOI allows for a normalization of the contribution from each gaze point. It also addresses the problems shown in fig. 3 . When a respondent is close, the covering of the Gaussian gaze point of the 3D AOI will be small with a locally high intensity. Respondents far away will cover a larger area in the 3D AOI, which will result in less locally intensive mapping. It also handles change in perspective, while effectively shaping the Gaussian according to the viewpoint without introducing parallax error. Since the sum of Gaussian gaze points is done on a 3D model, the heatmap can be projected into any frame or reference image. The backprojection of the a heatmap is shown in fig. 8(a) as an overlay to the original image.

\section{Results}

The core of our presented pipeline is the ability to correctly find the pose of the wearble gaze tracker relative to the 3D AOI in a given frame. Validating this after filtering puts each frame in one of four categories:

True positive correctly detecting the AOI.

True negative correctly not detecting the AOI.

False positive incorrect detection of the AOI.

False negative incorrectly not detecting the AOI.

Reviewing the output videos with 3D AOI overlay backprojected as presented in fig. 5(b) is an easy way to quickly asses the quality of the AOI detection. Such a review shows non or only a very few false positives, but some false negatives. Since the gaze tracker has a very small sensor, the sensor struggles with low indoor light, which results in both frames with motion blur from head movement and rolling shutter. In the supermarket setting, these frames provide the vast majority of false negatives and one could debate, whether they are actually false negatives. Occlusion from people or other shelves can also cause false negatives. Reviewing both the frame positions as a graph in fig. 6 or the resulting spatial positions in fig. 7(a) are can also provide quick qualitative verifications in addition to reviewing a video with backprojected 3D AOI. 


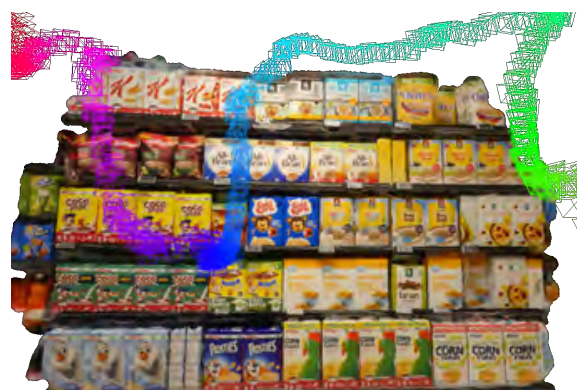

(a) Estimated poses

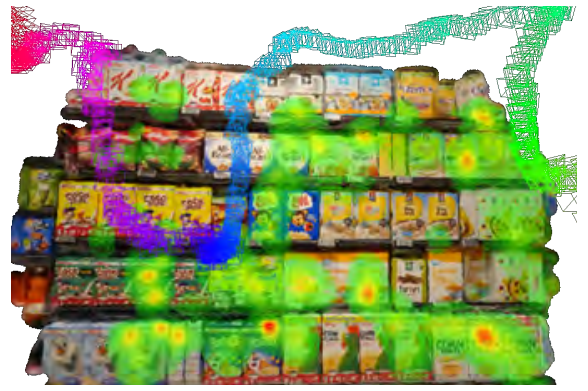

(c) Cereal heatmap

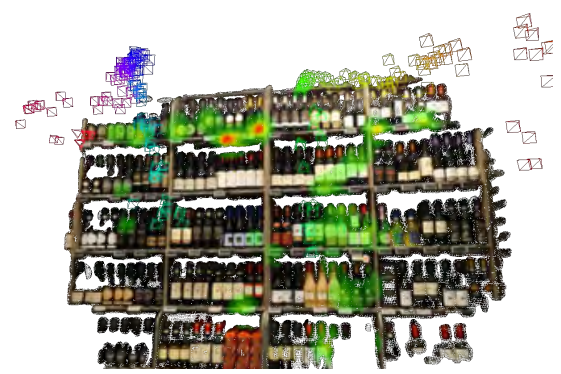

(e) Wine heatmap

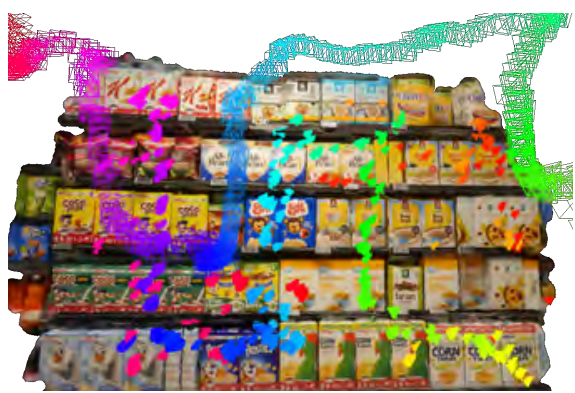

(b) 3D gaze points

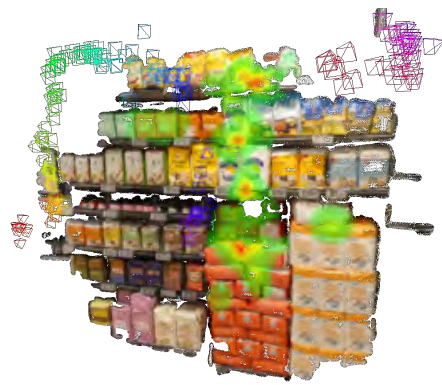

(d) Flour heatmap

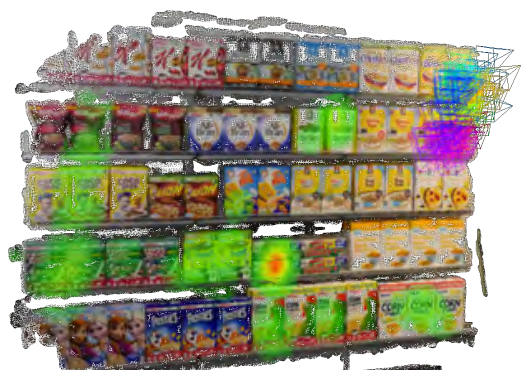

(f) Mock-up heatmap

Fig. 7. Poses, gaze points and heatmaps obtained from the data of the 5 sections included in our study.

We have reprojected the heatmap into a reference image, which has also been applied homography gaze mapping using the iMotions 6.2 software [4] and the results are shown in fig. 8 . The heatmaps are both based on raw data samples but using different techniques: 3D mapping and homography mapping respectively. This means they cannot be compared directly, however there have clear similarities of the path pattern and duration of attention. 


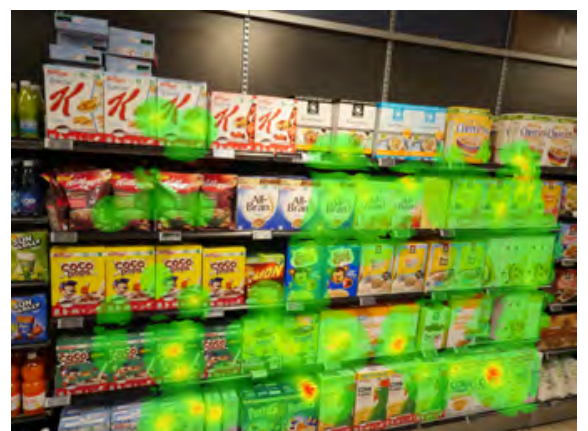

(a) 3D heatmap backprojected into reference image

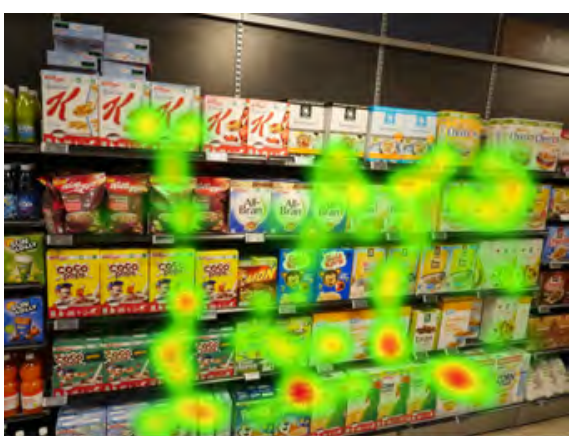

(b) 2D heatmap from iMotions software

Fig. 8. Heatmaps based on 3D gazemapping and 2D gazemapping. For the 3D mapping the heatmap has been backprojected into the DSLR frame used for the 2D mapping.

\section{Conclusion}

We have successfully created 3D AOIs and heat maps for respondents visiting the five sections in our data set: vegetables, cereal, flour, wine, and cereal mockup. Our proposed pipeline does away with the problems relating to mapping gaze using a homography. The proposed pipeline is fully automatic and runs at $\tilde{1}$ fps using a partially Matlab, mex, vlfeat and OpenCV. A full $\mathrm{C}++\mathrm{im}-$ plementation will provide further speed up, but the scope was to demonstrate a feasible pipeline, which allows researchers to spend time only on the results, once processed. Concerns such as visual attention mapped based on fixation vs. raw data, size of the Gaussian gaze point, and cross respondent analysis has not been evaluated. We found that our pipeline works well in in-store settings, since store products tend to have very distinct image features. However, settings with only repeating image features, such as frames with only the same product present, complicates the feature matching. This is often the case, when the respondent is very near a product shelf. On the other hand, detection works well in the case where the respondent is inspecting the shelves at an arm length distance, which in many cases is the important frames for generating heat maps. Our approach provides a fully automatic method of mapping gaze data and positioning of the respondent relative to the AOI, thus adding another dimension to the resulting data.

\section{Acknowledgements}

This work has been funded by the Innovation Fund (Denmark) and carried out in collaboration with iMotions. We would like to thank both for the support and collaboration. 


\section{Bibliography}

[1] G. Bradski. OpenCV. Dr. Dobb's Journal of Software Tools, 2000.

[2] Yasutaka Furukawa and Jean Ponce. Accurate, Dense, and Robust Multi-View Stereopsis. IEEE Transactions on Pattern Analysis and Machine Intelligence, 1(1):1-14, 2007. ISSN 01628828. doi: http://doi.ieeecomputersociety.org/10.1109/TPAMI.2009.161.

[3] Kenneth Holmqvist, Marcus Nyström, Richard Andersson, Richard Dewhurst, Halszka Jarodzka, and Joost Van de Weijer. Eye tracking: A comprehensive guide to methods and measures. OUP Oxford, 2011.

[4] iMotions. imotions biometric research platform, 2017. URL https://imotions.com/.

[5] Michael Kazhdan, Matthew Bolitho, and Hugues Hoppe. Poisson Surface Reconstruction. In Proceedings of the fourth Eurographics symposium on Geometry processing, SGP '06, pages 61-70, Aire-la-Ville, Switzerland, Switzerland, 2006. Eurographics Association, Eurographics Association. ISBN 3-905673-36-3.

[6] David G Lowe. Distinctive Image Features from Scale-Invariant Keypoints. International Journal of Computer Vision, 60(2):91-110, 2004.

[7] Michael Maurus, Jan Hendrik Hammer, and Jürgen Beyerer. Realistic heatmap visualization for interactive analysis of $3 \mathrm{~d}$ gaze data. In Proceedings of the Symposium on Eye Tracking Research and Applications, pages 295-298. ACM, 2014.

[8] Lucas Paletta, Katrin Santner, Gerald Fritz, Heinz Mayer, and Johann Schrammel. 3d attention: measurement of visual saliency using eye tracking glasses. In CHI'13 Extended Abstracts on Human Factors in Computing Systems, pages 199-204. ACM, 2013.

[9] Thies Pfeiffer. Measuring and visualizing attention in space with 3d attention volumes. In Proceedings of the Symposium on Eye Tracking Research and Applications, pages 29-36. ACM, 2012.

[10] Tobii Pro. Tobii pro glasses 2, $2017 . \quad$ URL http://www.tobiipro.com/product-listing/tobii-pro-glasses-2/.

[11] Christian Purucker, Jan R Landwehr, David E Sprott, and Andreas Herrmann. Clustered insights: Improving eye tracking data analysis using scan statistics. Psychological Considerations on Car Designs-An Investigation of Behavioral and Perceptual Aspects Using Eye Tracking and Cross-Cultural Studies, 2012.

[12] Bill Triggs, P McLauchlan, Richard Hartley, and A Fitzgibbon. Bundle Adjustment - A Modern Synthesis. Vision Algorithms: Theory and Practice, 1883:298-372, 2000. ISSN 0302-9743. URL http://www.springerlink.com/content/plvcrq5bx753a2tn.

[13] Andrea Vedaldi and Brian Fulkerson. Vlfeat. Proceedings of the international conference on Multimedia - $M M$ 
'10, 3(1):1469, 2010. doi: $10.1145 / 1873951.1874249 . \quad$ URL http://dl.acm.org/citation.cfm?doid=1873951.1874249.

[14] Zhengyou Zhang Zhengyou Zhang. Flexible camera calibration by viewing a plane from unknown orientations. Proceedings of the Seventh IEEE International Conference on Computer Vision, 1(c):0-7, 1999. ISSN 01628828. doi: 10.1109/ICCV.1999.791289. 